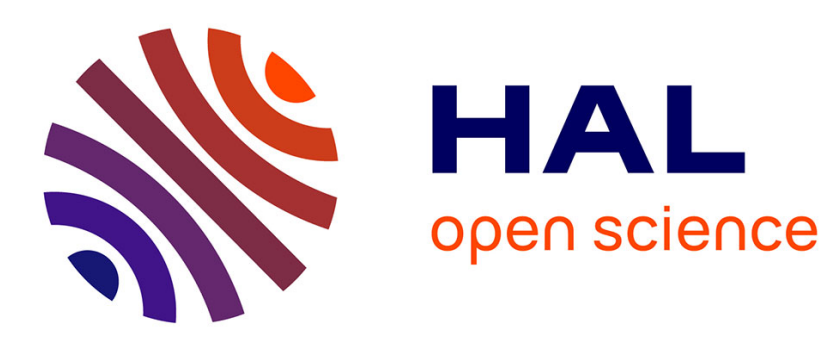

\title{
Humanisme Juridique et Science du Droit au XV Siècle
}

Patrick Gilli

\section{To cite this version:}

Patrick Gilli. Humanisme Juridique et Science du Droit au XV Siècle: tensions compétitives au sein des élites lettrées et réorganisation du champ politique. Revue de Synthèse, 2009, 130 (4), pp.571-593. 10.1007/s11873-009-0094-3 . hal-00545751

\section{HAL Id: hal-00545751 \\ https://hal.science/hal-00545751}

Submitted on 12 Dec 2010

HAL is a multi-disciplinary open access archive for the deposit and dissemination of scientific research documents, whether they are published or not. The documents may come from teaching and research institutions in France or abroad, or from public or private research centers.
L'archive ouverte pluridisciplinaire HAL, est destinée au dépôt et à la diffusion de documents scientifiques de niveau recherche, publiés ou non, émanant des établissements d'enseignement et de recherche français ou étrangers, des laboratoires publics ou privés. 


\title{
HUMANISME JURIDIQUE ET SCIENCE DU DROIT AU XV' SIÈCLE Tensions compétitives au sein des élites lettrées et réorganisation du champ politique
}

\author{
Patrick GILLI*
}

RÉSUMÉ: La question de l'humanisme juridique a souvent été traitée sous la forme des avancées méthodologiques apportées successivement par les découvertes philologiques. Les juristes convertis aux bienfaits de l'approche savante des textes auraient ainsi modifié à la fois leur technique exégétique et leur façon d'enseigner (le mos docendi). En partant des différentes questions soulevées par le retour ad fontes, l'article cherche à préciser la portée politique de l'implantation de la jurisprudentia cultior qui fut bien plus qu'une conquête philologique. Il s'agissait d'ajuster le droit aux nouveaux défis de la culture politique, notamment à la lente émergence de la souveraineté qui trouvera son accomplissement chez Bodin.

Mots-CLÉS : humanisme, Italie, culture juridique, Moyen Âge, intellectuels médiévaux.

\section{LEGAL HUMANISM AND SCIENCE OF LAW IN THE $15^{\text {th }}$ CENTURY}

Competitive tensions in the midst of learned elites and the reorganisation of the political field

ABSTRACT: The question of legal humanism was often presented in the shape of the methodological progress brought successively by philological discoveries. The jurists converted to the blessings of the "learned approach" of texts would have modified at the same time their exegetical analysis and the way they taught (mos docendi). Considering several questions raised by the return ad fontes, the article tries to clarify the political scope of the setting-up of the jurisprudentia cultior which was much more than a philological conquest. It was a matter of fitting the law for the new challenges of the political culture, in particular for the slow emergence of the sovereignty which will find its fulfillment with Bodin.

KEYWORDS: humanism, Italy, legal culture, intellectuals in the Middle Ages.

\footnotetext{
* Patrick Gilli, né en 1961, est professeur d'histoire du Moyen Âge à l'université de Montpellier III et vice-président de l'université en charge de la recherche. Ses travaux portent sur l'histoire des doctrines juridiques et politiques de la fin du Moyen Âge et de la première Renaissance. Il a récemment dirigé le recueil Les Élites lettrées au Moyen Âge. Modèles et circulation des savoirs en Méditerranée occidentale. $X I{ }^{e}-X V^{e}$ siècles (Montpellier, Presses universitaires de la Méditerranée, 2008), publié «Imperium et Italie au $\mathrm{Xv}^{\mathrm{e}}$ siècle: juristes et humanistes face à la dé-romanisation de l'empire » (Rechtsgeschichte. Zeitschrift des Max-Planck-Instituts für europäische Rechtsgeschichte, $\mathrm{n}^{\circ} 13,2008$ ) et en collaboration avec Julien Thery, Le Gouvernement pontifical des villes au temps de la théocratie, fin $X I{ }^{e}-m i-X I V^{e}$ siècle (Montpellier, Presses universitaires de la Méditerranée, 2009).

Adresse: Université Paul-Valéry, Route de Mende, F-34099 Montpellier cedex.

Courrier électronique: Patrick.gilli@univ-montp3.fr
} 


\section{JURISTISCHER HUMANISMUS UND RECHTWISSENSCHAFT IM 15. JH. \\ Konkurrenzspannungen innerhalb der literarischen Eliten und Neuorganisation des politischen Feldes}

Zusammenfassung: Die Frage des juristischen Humanismus ist häufig unter dem Aspekt der schrittweise durch die philologischen Entdeckungen erbrachten methodologischen Fortschritte behandelt worden. Die zu den Wohltaten der gelehrten Herangehensweise konvertierten Juristen hätten demnach ihre exegetische Technik und ihre Art des Ausbildens (den mos docendi) zugleich verändert. Ausgehend von anderen, durch die Rückkehr ad fontes aufgeworfenen Fragen, versucht der Artikel, die politische Tragweite der Einführung der jurisprudentia cultior präzise zu bestimmen, die sehr wohl mehr als eine philologischer Eroberung war. Es handelte sich um eine Anpassung des Rechtes an neue Herausforderungen der politischen Kultur, insbesondere an die allmähliche Herausbildung der Souveränitat, die ihre Erfüllung bei Bodin finden sollte.

STICHWÖRTER: Humanismus, Italien, juristische Kultur, Mittelalter, mittelalterliche Gelehrte.

باتريك جيلي : إنسانية، قانو نية و علم القانون في القرن 17 . ضغوطات متنافسة وسط النخب الأدبية و إعادة تنظيم المجال السياسي.

ملّخص : غالبا ما تدرس مسألة الإنسانية القانونية عبر تسبيقات منهجيَّة أفرزتها بالتَّو الي الإكتشافات

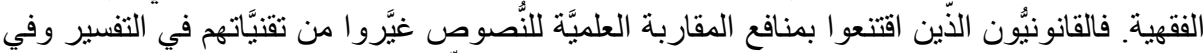

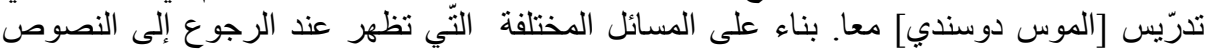

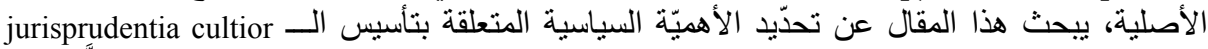

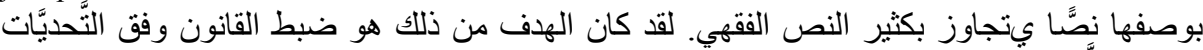

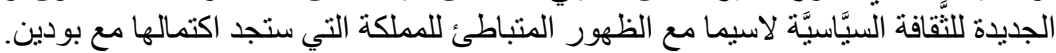
الكلمات المفاتح : إنسانية، إيطاليا، ثقافة قانونية، القرن الوسيط، المثققون القروسطيون.

法的人文主義と 15 世紀における法科学

学識エリートらの競争緊迫と政治分野での再構成郘ック・ジリ

要約: 法的人文主義の問題は、文献学的発見によって徐々にもたらされた方 法論的突出部として取り扱われがちである。知識人の文書に取り扱い方に恩 恵を浴した法学者らは、彼らの注釈の技術と教授法（mos docendi）を同時 に変更した。本文は、原点に立ち返ることによって浮き彫りにされる様々な 問題から出発し、単なる文献学的試み以上である法律的認識 (jurisprudentia cultior）を導入した政治的結論について取り上げたい。つまり、ボダンにお いて実現した至上権の出現のような政治文化における新しい抵抗に対し法律 を実行することである。

キーワード：人文主義、イタリア、法的認識、中世、中世知識人 
$\mathrm{L}$ a question de l'humanisme juridique est de celles qui ont bénéficié ces dernières années d'un renouvellement historiographique grâce aux recherches italiennes, anglo-saxonnes ou allemandes ${ }^{1}$. Toutefois ces études sont davantage centrées sur les aspects philologiques ou techniques du mouvement que sur la portée culturelle et politique d'un phénomène qui a mis en jeu non seulement la qualité des textes disponibles mais encore la place, dans le périmètre des pouvoirs de l'état, de ceux qui les interprétaient. La définition de ce concept d' «umanesimo giuridico», de «legal humanism » ou, pour reprendre un terme plus historique, de «jurisprudentia elegantior [ou] cultior » n'est pas des plus aisée et peut se concevoir à des niveaux très différents ${ }^{2}$. On évoquera ici les diverses facettes de cette question pour suggérer les problématiques que le sujet recèle, sous bénéfice d'inventaire, en mettant l'accent sur la situation italienne des Trecento et Quattrocento, dans les turbulences de la naissance de ce mouvement culturel de rupture que fut le premier humanisme. Mouvement de rupture, disons-nous, car le paradigme humaniste ne se réduit pas à la redécouverte des textes antiques, à leur traitement philologique et aux commentaires des Anciens. Il s'agissait bien plus profondément de proposer une organisation de la connaissance qui prenait à rebours l'arborescence ordinaire des disciplines et la démarche universitaire dans son ensemble ${ }^{3}$. Cela passait par le développement d'institutions d'enseignement extra-universitaires, par le dialogue critique avec les auctoritates (autorités au sens intellectuel). De ce point de vue, les récents travaux visant à insérer les studia humanitatis dans une création continuée depuis le milieu du XIII ${ }^{\mathrm{e}}$ siècle, réduisant de facto les effets et les efforts de rupture, nous semblent discutables ${ }^{4}$. Que l'humanisme ne soit pas sorti tout armé de la tête de Pétrarque, personne ne saurait le contester; qu'il ait été fécondé par la richesse des artes dictaminis (manuels de rédaction des actes et des lettres) et l'extrême inventivité des maîtres d'école de rhétorique dont l'héritage a perduré longtemps encore au XIV siècle, voilà qui a été largement démontré ${ }^{5}$. Que l'on puisse baptiser d'humaniste toute importation raisonnée et réinterprétée des sources et des techniques d'écriture antiques dans la culture contemporaine passe à la rigueur. Toutefois, tirer prétexte de ces dialogues

1. La part de l'historiographie française dans ces débats est modeste: pour un bilan des opinions courantes en France sur le phénomène, voir ThIREAU, 2003. Toutefois, une place à part revient aux travaux d'ARABEYRe, 2003 et, sur un point particulier, 1999. À noter, la récente publication du colloque Droits et justice dans l'Europe de la Renaissance (voir PitTion, éd., 2009) qui apporte quelques éclairages complémentaires.

2. Voir à titre synthétique, OsLer, 2001.

3. BotTin, 1982.

4. Le véritable fondateur de cette réécriture historiographique de l'humanisme est Ronald WITT, 2000. Dans ce maître-ouvrage, le savant américain s'employait à montrer que les soubassements intellectuels des studia humanitatis étaient déjà présents dans les cités du Nord de l'Italie, notamment Padoue, que Pétrarque ne représentait somme toute que la troisième génération d'humanistes italiens et que l'apport essentiel de ce dernier a résidé prioritairement dans la christianisation de la redécouverte des écrits antiques.

5. BLACK, 2001, insiste sur la continuité des textes d'apprentissage du latin dans les écoles en Italie entre les XIII ${ }^{e}$ et les $\mathrm{XV}^{\mathrm{e}}$ siècles, mais pointe toutefois un aspect essentiel dans la différenciation sociale qui se joue avec l'humanisme au milieu du XIv ${ }^{\mathrm{e}}$ siècle entre les grammatici et les auctoristi (professeurs enseignant le latin à partir des grands auteurs et non plus à partir des manuels d'apprentissage que pratiquaient les grammatici). L'enjeu pour les plus habiles et les plus ambitieux de ces maîtres d'école (qui allaient former la trame professionnelle de ceux que l'on appelle les humanistes) était de rehausser leur statut social en rehaussant leurs niveaux de compétences et de maîtrise savante. 
continués et renouvelés avec les Anciens pour réduire la rupture humaniste introduite par Pétrarque à une posture d'intellectuel compulsivement autobiographique qui surjoue son rôle ${ }^{6}$ mérite d'être débattu. La discussion autour de la scientia legalis (plus que tout autre secteur disciplinaire) permet de prendre la mesure de la tension critique qui secouait les intellectuels italiens et de la volonté de poser une alternative dans les hiérarchies du savoir, alternative que nous proposons d'appeler le paradigme humaniste. Par l'ampleur et la portée de la démarche, c'est à une redistribution des rôles sociaux et de la place politique des élites lettrées tardo-médiévales que conviaient les humanistes postpétrarquiens. Il n'est pas question ici de nier la prise de risque intellectuelle des lettrés padouans, ni leur singularité en leur temps, c'est-à-dire dans les premières décennies du Trecento. Mais ce que le débat autour du droit permet d'éclairer, à partir du Lauréat, c'est précisément le changement d'objectif politico-culturel de cette nouvelle esthétique et de cet effort de refondation de la connaissance. Les postures, les gestes symboliques, les autopromotions n'en sont pas absents, mais ce que nous voudrions montrer ici c'est que la question du droit, en Italie, touchait au fonctionnement politique des régimes communaux et post-communaux et à la place des juristes en leur sein, fonction à nulle autre pareille en Europe. En examinant ensuite comment s'est diffusée une méthodologie humaniste appliquée aux textes juridiques, nous voudrions aussi montrer les effets politiques induits par cette mise en cause des sources faisant jusqu'alors autorité.

\section{LES ORIGINES DE L'HUMANISME JURIDIQUE: LA DÉSACRALISATION DE LA SCIENTIA LEGALIS}

À plusieurs reprises, Pétrarque a croisé le fer contre les disciplines académiquement reconnues, dans un effort acharné pour déclasser ces savoirs fraîchement, mais solidement, constitués et leurs représentants (juristes et médecins en particulier). On ne trouve pas avant Pétrarque, tout particulièrement chez ceux que Ronald Witt considère comme chefs de file des deux premières générations d'humanistes, un quelconque engagement dans la critique des disciplines savantes. C'est là une ligne de fracture essentielle qui distingue le moment pétrarquien de ses prodromes. Au travail d'écriture poétique d'un Lovato Lovati ou d'un Albertano Mussato, qui devaient surtout prendre position face à la domination culturelle de la poésie franco-provençale en Italie et revaloriser la fonction de poésie dans le champ de la connaissance autour d'une nouvelle esthétique ${ }^{7}$, succède l'approche de Pétrarque. Tout en ayant à cœur de valoriser la veine culturelle italienne ${ }^{8}$, dont la réappropriation de l'héritage antique était la pierre angulaire, cette approche vise à instaurer une contre-hiérarchie intellectuelle et institutionnelle ${ }^{9}$ qui ferait pièce à la domination écrasante des doctores legum dans les

6. WiтT, 2000, p. 173.

7. Récemment WiTt, 2008, est revenu sur la question des premières générations d'humanistes, en montrant que les références à la littérature classique et aux techniques poétiques issues de cette nouvelle esthétique touchaient également les historiens laïques des communes italiennes.

8. Gagliano, 2007.

9. À cet égard la Collatio laureationis, c'est-à-dire le discours que prononça Pétrarque lors de la remise du laurier poétique à Rome en 1341, peut se lire comme un anti-modèle du discours d'intronisation des docteurs universitaires lors du conventus (la cérémonie de remise des insignes doctoraux). 
sociétés urbaines italiennes. Il est d'ailleurs frappant que Lovato Lovati, l'un des seuls poètes contemporains de Pétrarque que ce dernier cite avec respect, se voit précisément reprocher par ce même Pétrarque de n'avoir pas eu le courage de se séparer du droit et de ses techniques :

«Récemment Lovato de Padoue aurait pu très facilement être le prince de tous les poètes que notre époque et celle de nos pères a portés, si ayant embrassé l'étude du droit civil, il n'avait mêlé les Douze Tables aux Neuf Muses et rabaissé son esprit des soucis célestes au bruit des tribunaux ${ }^{10}$. »

C'était clairement signifier que le chemin n'était qu'à moitié parcouru... et qu'il revenait à Pétrarque de le continuer. Il importe de ne pas se méprendre sur le sens de la polémique et du débat: l'enjeu n'est rien moins que la redéfinition du champ du savoir et de la place des lettrés d'un nouveau genre dans la nouvelle économie de la connaissance. Rien d'étonnant à ce que les cibles qui, de Pétrarque à Valla (autrement dit de la période d'affirmation de ce nouveau paradigme à celle de son institutionnalisation), ont été le plus visées furent les juristes. Le proto-humanisme padouan était porté par des notaires et des juges, et n'attaquait pas frontalement le groupe dominant des doctores legum. L'humanisme revendicatif d'un Pétrarque se donnait comme objectif de fonder de nouvelles hiérarchies, plaçant au sommet évidemment une figure inédite de lettré, l'humaniste, intellectuel organique de l'oligarchie des régimes seigneuriaux ${ }^{11}$, comme le notaire était l'intellectuel organique des régimes communaux.

Dès les débuts des critiques contre le droit à l'initiative de Pétrarque, plusieurs thèmes se sont mêlés pour mettre à mal son enseignement, sa méthodologie et sa finalité sociale. Ces dénonciations ont fait l'objet d'un travail précédent ${ }^{12}$, mais il semble utile cependant de revenir sur certains thèmes qui portaient sur le cœur même de la dogmatique juridique propre au droit commun. Il était admis que le ius commune était à la fois un ensemble doctrinal qui s'appuyait sur le corpus justinien augmenté du corps du droit de l'Église ${ }^{13}$, et un système d'organisation juridique et judiciaire qui laissait aux juristes un espace de créativité considérable par le jeu de l'interpretatio iuris (interprétation $\mathrm{du}$ droit) ${ }^{14}$. Lorsque apparaissent les critiques les plus percutantes contre la tradition juridique sous la plume des humanistes - et nous laissons de côté les topoi classiques sur la vénalité des juristes et des hommes de loi, les prétentions de ces derniers à une noblesse ex scientia (différente de la noblesse de robe de tradition française); nous laissons même de côté la dénonciation du latin barbare des juristes -, l'objet le plus sensible de ce faisceau de dénonciations ou d'imprécations fut la prétendue universalité

10. PÉtrarque, 1943, p. 84: «Lovatus patavinus fuit nuper poetarum omnium quos nostra vel patrum nostrorum vidit etas facillime princeps, nisi iuris civilis studium amplexus et Novem Musis duodecim tabulas immiscuisset et animum ab eliconis curis ad forensem strepitum deflexisset. »

11. Il est frappant que les plus grands humanistes depuis Pétrarque aient posé comme geste inaugural de leur carrière l'abandon des études juridiques. Il s'agit là d'une posture revendiquée, presque un rituel d'intégration, qui scelle une sodalitas.

12. Gilli, 2003.

13. Sur la définition du ius commune, voir notamment Mayali, 1999; Giordanengo, 1999.

14. Cortese, 1982. 
des lois romaines. Bruni, Le Pogge, d'autres encore, reprirent cette idée d'un strict localisme du droit romain, faisant voler en éclats les revendications juridiques des glossateurs et des commentateurs à incarner ou à faire vivre la ratio scripta, forme de savoir métahistorique, transcendante et universelle ${ }^{15}$. En pointant du doigt toutes les zones européennes ou mondiales de non-romanité juridique ${ }^{16}$, en mettant aussi en évidence la diffusion d'un ius proprium (droit propre) en Italie qui se démarquait du ius commune (droit commun) ${ }^{17}$, au moment d'ailleurs où les ordonnancements juridiques des villes italiennes étaient remis en question par la création des états territoriaux qui nécessitaient des réponses précises sur l'articulation entre les différents droits locaux, les humanistes indiquaient la faille constitutive du ius commune qui n'apparaissait plus que comme une construction idéologique historiquement datée: bref, le droit romain à la Renaissance n'avait rien à voir avec la renaissance du droit romain, comme la voyaient les juristes. La réintroduction des textes justiniens au XII siècle s'était donnée une ambition grandiose: remettre en mouvement un texte pluriséculaire en suggérant sa parfaite actualité juridique et sa totale congruence avec la société du temps, alors que les auteurs des $\mathrm{XV}^{\mathrm{e}}$ et $\mathrm{XVI}{ }^{\mathrm{e}}$ siècles redimensionnaient le travail accompli par leurs prédécesseurs des $\mathrm{XII}^{\mathrm{e}}$ et $\mathrm{XIV}^{\mathrm{e}}$ siècles en rappelant les limites de l'opération: la valeur sacrée des leges et celle de leurs desservants zélés en sortaient singulièrement diminuées. La montée en puissance du groupe professionnel des juristes en Italie dès le $\mathrm{XII}^{\mathrm{e}}$ siècle s'était en effet accompagnée d'un effort constant pour donner à la culture des civilistes une dimension quasi religieuse (les docteurs en droit étant dits «prêtres de la loi »), conférant aux juristes une conscience de soi extrêmement valorisante, à la hauteur, du reste, de la place sociale qui était la leur dans les sociétés urbaines italiennes. Les attaques dont ils faisaient l'objet à partir $\mathrm{du} \mathrm{XIV}^{\mathrm{e}}$ siècle visaient précisément à réduire ces prétentions sur la valeur quasi sacrée de leur mission et sur les textes où ils enracinaient leurs connaissances. Cette désacralisation de la scientia legalis, réduite aux yeux de ses détracteurs à un savoir-faire farineux, constitue le premier défi des humanistes à la science juridique.

L'historien se doit d'être sensible à la chronologie des accusations car elle est loin d'être innocente: si l'on regarde le moment le plus intense de la polémique entre juristes et humanistes, une fois passées les premières salves pétrarquiennes, on constate qu'il se déploie peu après la grande crise des pouvoirs universels, à savoir dans les années 1430 ou 1440, comme si une « fenêtre de tir » s'était ouverte, autorisant le déploiement d'arguments particulièrement virulents, alors que la papauté tente péniblement de se réorganiser sous Eugène IV: c'est de cette période que datent les textes d'un Valla, d'un Piccolomini ou d'un Alberti ou d'un Raimondi ${ }^{18}$. On ne retrouvera pas jusqu'au

15. On remarquera, entre Pétrarque et Bruni, un grand vide; la génération 1370-1400, dominée par la stature de Coluccio Salutati (un notaire de formation!) n'a pas digéré immédiatement toutes les audaces de Pétrarque, sur ce sujet comme sur bien d'autres.

16. Toutes les citations sur le sujet sont extraites de GilLI, 2003, passim, et MAFFeI, 1972.

17. Voir par exemple les remarques ironiques de la lettre de Leonardo Bruni adressée à un jeune aristocrate florentin au sujet de cette variabilité locale des lois dans GARIN, 1947, p. 8: «Praeterea bonitas et virtus stabilis est, ius autem locis et temporibus variatur, ut saepe quod Florentiae legitimum est, Ferrariae sit contra legem.»

18. GILLI, 2003. 
$\mathrm{XVI}^{\mathrm{e}}$ siècle des discours aussi forts que ceux rédigés contre le droit romain durant ces décennies. Moins que d'humanisme juridique, il faut parler alors d'une dénonciation totale des principes mêmes de la sapientia legalis (sagesse du droit). C'est à un véritable travail de sape que se livrent les humanistes italiens pour qui l'activité juridique est incompatible avec la nouvelle hiérarchie intellectuelle en cours de construction. Précisons toutefois que, très tôt, des initiatives encore balbutiantes furent entreprises par des lettrés frottés de culture juridique pour historiciser le droit antique et tenter de lui redonner la profondeur chronologique et l'ancrage contextuel qui lui faisaient défaut. C'est ainsi que dès 1425, Andrea di Domenico Fiocchi, à la fois juriste et élève du savant grec Chrysoloras, rédigeait son De potestatibus Romanis où il proposait une histoire des magistratures et des prêtrises romaines ${ }^{19}$. Peu de temps après, c'était autour de Francesco Filelfo de proposer, en 1439, la première compilation historique des lois antiques dans une lettre à un patricien vénitien (Epistula ad Cornelium $^{20}$ ).

Mais si humanisme juridique il y eut, il fut d'abord une réponse des juristes aux initiatives et aux canonnades humanistes ${ }^{21}$ : Pietro del Monte en constitue une figure notable, même si l'on reste frappé par la disjonction entre son activité d'humaniste dont témoigne sa collection de lettres personnelles et sa production théorique de canoniste beaucoup plus traditionnelle ${ }^{22}$. Plusieurs juristes de cette génération donnent le sentiment d'avoir été partagés par une sorte de schizophrénie intellectuelle entre les deux tendances culturelles. Il n'est pas indifférent de noter qu'un des premiers travaux véritablement humanistes entrepris par un juriste fut l'œuvre d'un Catalan, formé à Bologne: Joan Ramon Ferrer, docteur in utroque en 1451, lequel rédigea en 1448 un dialogue censé s'être tenu à Bologne entre un certain Antonio de Sicilia et lui-même, sur le thème de l'antiquité des lois. Le motif en était le suivant: Antonio défend la thèse avancée par Accurse et Bartole selon laquelle l'essentiel des lois du Digeste ont été rédigées avant la naissance du Christ; Joan Ramon, au contraire, affirme que l'on peut déduire de nombreux auteurs classiques, par une méthode en quelque sorte génétique, que la plupart des lois sont postérieures au Christ. Les juges de cette dispute publique sont deux juristes bolonais de renom : Antonio de Pratovecchio et Scipion de Gozzadini $^{23}$. Si la facture de l'ensemble est convenue (un dialogue pro et contra qui s'apparente plus à une disputatio académique qu'au dialogue de type humaniste ${ }^{24}$ ), les arguments en revanche sont empreints de cette atmosphère de changement qui devait commencer à se diffuser parmi la jeunesse universitaire italienne du milieu du Quattrocento, friande de nouveautés. C'est ainsi que le juriste barcelonais met en cause les principales autorités de l'enseignement du droit, Accurse et Bartole. Même s'il prend soin de reconnaître leurs mérites, il n'hésite pas à pointer les limites de leurs

19. LAUREYS, 1995.

20. FerRary, 1995.

21. Le tableau le plus significatif sur la place idéale qui devrait revenir aux juristes et aux humanistes dans le gouvernement des sociétés se trouve chez l'humaniste lombard Cosma Raimondi : voir GiLLI, 1998; le texte est un catalogue presque exhaustif de tous les arguments en faveur d'une supériorité politique des humanistes (oratores, selon la terminologie de Raimondi) sur les juristes.

22. Quaglioni, 1984; sur l'activité humaniste de Pietro del Monte, voir Rundle, 2004.

23. FERRER, 2006.

24. Marsh, 1980, et plus récemment, Paternoster, 2000. 
connaissances ${ }^{25}$. Les auteurs invoqués sont ceux du catalogue humaniste: Sénèque, Cicéron, mais aussi Giovanni Lamola (qualifié de noster communis doctissimus humanissimusque praeceptor) ou Guarin de Vérone. Cet opuscule de jeunesse ${ }^{26}$ constitue l'un des plus précoces témoignages de l'intérêt naissant des juristes bolonais envers les nouveaux savoirs et, dès le début, glossateurs comme commentateurs sont pris pour cible. Bien sûr, l'effort est encore modeste, mais il méritait d'être rappelé comme une des premières réponses issues du milieu juridique lui-même au défi humaniste. Nous sommes loin, assurément, des audaces d'un Valla ou d'un Alberti au milieu du siècle, lesquels n'eurent pas, non plus, une postérité immédiate. Il faut, en effet, en convenir: par la suite, sans disparaître, la polémique baisse d'un cran et, disons-le, d'intensité intellectuelle ${ }^{27}$. À cela plusieurs raisons. D'abord, les juristes italiens ont tardé à relever le défi posé par le nouveau paradigme culturel: les sollicitations viennent le plus souvent des humanistes pour un examen plus serré des sources manuscrites (et l'on pense bien sûr aux initiatives du Politien sur la littera florentina ${ }^{28}$ et à celles du juriste Bolognini ${ }^{29}$ ). Ensuite l'institutionnalisation de l'humanisme ${ }^{30}$, c'està-dire la conquête des chaires universitaires par les humanistes et l'implantation du mouvement dans les cours seigneuriales ${ }^{31}$ incitaient à la modération et à la réitération peu inventive des critiques, d'autant que la reprise en mains par la papauté d'une

25. À titre d'exemple, voici comment Ferrer juge de la connaissance de l'histoire des grands juristes de son temps (FERRER, 2006, p. 132): «Sed quamquam iste noster Accursus in historias cognoscendas summam operam et studium contulisset, non tamen permirum esse deberet eum his in rebus defecisse, quemadmodum nulla admiratione afficimur, etiam si Servium aliosque doctissimos, qui de historiis accurate scripserunt, multis in locis errore quodam falsum cum vero miscuisse palam fit. Ac etiam quemadmodum remur non esse admiratione dignum quamvis, non modo ipse Accursius, sed etiam Cinus, Bartolus, Baldus aliique permulti, qui post et auctoritatis magnitudine et scientiarum amplitudine praestiterunt, multis in rebus, ad ius dumtaxat spectantibus, a veritate quam plurimum abhoruisse videantur. » (Mais, quoique notre Accurse ait apporté la plus grande attention et le plus grand soin à la connaissance de l'histoire, il ne serait pas très étonnant qu'il ait eu des lacunes en ce domaine, de la même manière que nous ne sommes pas étonnés qu'il est évident que Servius et d'autres hommes très doctes qui ont écrit avec soin des récits historiques ont mêlé en maints endroits le faux et le vrai par erreur. De même, je pense qu'il n'est pas étonnant que non seulement Accurse, mais aussi Cinus, Bartole, Balde et beaucoup d'autres qui par la suite se sont illustrés par la grandeur de leur autorité et l'ampleur de leur science, semblent s'être éloignés grandement de la vérité, y compris en ce qui concernait le droit.)

26. La date de naissance de Ferrer n'est pas connue avec précision mais se situe vers 1415 . À côté de ce travail de " proto-humanisme juridique », notre savant a pratiqué la poésie (latine et vernaculaire) et les traités de grammaire latine (dont un ouvrage sur les pronoms); il n'a pas négligé pour autant l'activité juridique en laissant trois volumes de consilia. Il meurt en 1490.

27. À titre d'exemple, voir OsLer, 1990.

28. Ainsi dénommait-on le manuscrit supposé le plus ancien du Digeste de Justinien, longtemps conservé à Pise (où on l'appelait la Littera pisana), puis, lorsque Pise fut conquise par Florence en 1406, conservé au Palais de la Seigneurie à Florence (de là, le nom de Littera florentina). Sur les usages de ce manuscrit au Xve siècle, voir GiLli, 2008.

29. CAPRIOLI, 1969. Voir aussi AsCHERI, 1977 et 1998.

30. Sur ce phénomène, voir GILLI, 2007.

31. Une enquête d'ensemble manque toujours, mais des études locales montrent clairement le mouvement d'ouverture de chaires de studia humanitatis le plus souvent d'ailleurs à l'initiative des autorités publiques: CORTESE, 1985, rapporte comment le roi Ferrant le fit pour l'université de Naples en 1465; pour d'autres exemples, voir CORTESE, 1995, vol. II, p. 463 ; récemment LinEs, 2006, a proposé un survol plus global de la question. 
partie non négligeable des humanistes à qui la curie offrait des débouchés notoires n'autorisait plus guère les mises en cause féroces du droit canon, comme avait tenté de le faire Valla ${ }^{32}$. N'oublions pas, en effet, que si l'humanisme juridique demeure essentiellement centré sur la renovatio des Pandectes, les critiques originelles n'excluaient pas le droit canon, encore que pendant tout le Quattrocento les attaques demeuraient ponctuelles et chantournées ${ }^{33}$.

Autrement dit, le climat intellectuel change dans les années 1460, mais l'on ne peut pas encore parler d'humanisme juridique. La confrontation cède la place à une sorte de routine dans la répartition des rôles sociaux de chacune de ces catégories de lettrés. Pourtant deux phénomènes conjoints vont modifier la donne. D'abord, l'humanisme italien devenant le paradigme dominant de la culture, les juristes ne peuvent pas l'ignorer totalement. Nous verrons jusqu'où ils peuvent aller sans risque sur les chemins ouverts par la critique des sources et le retour ad fontes (aux sources). D'autre part, cet espace de créativité qui était le leur dans l'interprétation des statuts, dans l'intervention judiciaire, dans l'élaboration d'une doctrine qui trouvait son application dans la pratique ordinaire, se clôt progressivement sous l'effet de la pression des états régionaux, en particulier des tribunaux d'appel dont les decisiones et les sententiae deviennent de plus en plus enseignées dans les studia juridiques et considérées comme sources de la jurisprudence au détriment des avis d'experts ${ }^{34}$. Cette évolution est capitale dans la naissance de l'humanisme juridique, qui ne saurait être interprétée comme un pur phénomène culturel, mais qui est aussi une réponse à la crise des ordonnancements institutionnels ${ }^{35}$. Les docteurs, et particulièrement les docteurs italiens, voyaient leur monopole culturel et social sévèrement mis en question.

\section{QUELLES ACTIVITÉS RECOUVRE L'HUMANISME JURIDIQUE?}

Bien sûr, il faut rappeler que l'humanisme, c'est avant tout le retour aux sources. Encore faut-il s'entendre sur le sens de ce retour. Il ne suffit pas seulement de transformer l'intellectuel en philologue pour en faire un humaniste, l'enjeu est infiniment plus profond: le retour aux sources signifie surtout une volonté de retrouver l'unité perdue du savoir quand le droit n'était pas dégagé de la grammaire et de la rhétorique et qu'il appartenait, en sous-ordre, à la sciencia sermocinalis (la science du discours) ${ }^{36}$. Ce dégagement du droit de l'unité originelle du savoir a produit aux yeux des humanistes des effets catastrophiques: le latin s'est corrompu, le texte fondateur a été enseveli sous des tombereaux de gloses ${ }^{37}$. Mais d'un point de vue plus pragmatique, dans l'ordre judiciaire, il a eu aussi des effets négatifs dont les juristes eux-mêmes sont

32. FUBINI, 1990 et 1991.

33. Quaglioni, 2004 et 2000; Rossi, 1999.

34. SAVELli, 1994; Gilli, 2005.

35. Voir les remarques de Garancini, 1990, et surtout Orestano, 1987, « La storia come problema centrale dell'umanesimo giuridico », p. 138-202.

36. Sur la naissance du droit comme science, voir ERRERA, 2003.

37. Tel est le sens de ce que Maurizio Manzin a appelé d'une formule provocante le « pétrarquisme juridique » : voir MANZIN, 1994. 
conscients. L'accumulation de gloses, le recours surtout à l'argument ab auctoritate des docteurs qui fait que dans les tribunaux, on ne juge plus nécessairement sur le texte de la loi, mais sur l'interprétation qu'en font les docteurs, tout cela fait entrer en crise le fonctionnement judiciaire (du moins en dénonce-t-on rituellement les dysfonctionnements), mais aussi la science juridique qui a tendance à s'ossifier et à se fossiliser. Le triomphe du bartolisme au $\mathrm{Xv}^{\mathrm{e}}$ siècle, dans sa version appauvrie car dépourvue de l'ampleur de vues de Bartole ou de Balde, aboutit à une sorte de droit des docteurs: c'est le moment où domine la communis opinio doctorum, qui n'est rien d'autre que la jurisprudence des docteurs ${ }^{38}$. Moment important que le développement de cette communis opinio doctorum comme tentative de sauvetage de la place des docteurs dans l'économie des savoirs et dans celle des pouvoirs en faisant d'eux les mètres étalons dans la jurisprudence et dans le travail des tribunaux. On aurait tort d'imaginer qu'il ne s'agit que d'un problème italien: les grandes monarchies européennes, Espagne et France en particulier, cherchent elles aussi à canaliser ce ius commune. En Espagne, au $\mathrm{XV}^{\mathrm{e}}$ siècle, on limitera les risques de débordements interprétatifs par l'instauration de l'opinio Bartoli comme droit subsidiaire ${ }^{39}$. En France, même si la situation est moins tendue en raison du rôle déjà important dans l'élaboration d'un droit royal par le Parlement, une tentative de dépassement du ius commune vient des efforts pour normaliser et harmoniser le droit coutumier (ordonnance de Montils-lès-Tours de $1454^{40}$ ). C'est tellement vrai qu'au Xvi ${ }^{\mathrm{e}}$ siècle, lorsque Cujas parle de ius commune, il entend non plus le droit romano-canonique, mais le droit coutumier et royal appliqué en France.

C'est dans ces conditions que s'élaborent les premières tentatives que l'on baptisera d'humanisme juridique. À partir du défi lancé par les humanistes, défi accru par la défiance que la tradition juridique suscitait désormais, il fallait trouver les solutions herméneutiques qui permettraient de conserver au juriste sa position centrale à la fois dans les rôles sociaux et dans l'architecture des savoirs: jurisconsultus, homo perfectus. L'effort a porté sur la méthodologie du droit et la méthodologie de l'enseignement: mos italicus ius docendi (manière italienne d'enseigner le droit) contre mos gallicus ius docendi (manière française d'enseigner le droit) ${ }^{41}$. Cette concentration sur la pédagogie autant que sur la méthodologie est véritablement humaniste. Pas de refondation méthodologique sans refondation pédagogique ${ }^{42}$ : lorsque le mouvement avait revêtu une dimension plus institutionnelle (accès aux chancelleries, ouverture d'écoles,

38. Voir les remarques de Rossi, 1999.

39. Cortese, 1995, vol. II, p. 459, n. 8.

40. Sur ce point, voir RigAUDIÈRE, 2004.

41. Bien évidemment, dès lors que l'on regarde de près le contenu des enseignements, on constate que l'opposition binaire entre les deux mores docendi est loin d'épuiser la réalité des pratiques pédagogiques, que les maîtres usent de tout un spectre méthodologique sfumato dans leurs cours. Voir ARABEYRE, 2008, qui retrace le passage de la repetitio classique à la repetitio « humaniste » jusqu'à la systématisation de l'enseignement du droit.

42. La plupart des grands juristes humanistes $d u X^{e}{ }^{e}$ siècle ont rédigé des traités sur la façon d'enseigner le droit: François Le Douaren, De ratione docendi discendique iuris (1554); Claudius Cantiuncula, De ratione studii legalis paraenesi (1522); Giulio PACE, De iuris civilis difficultate ac docendi methodo (1586). Voir CARPINTERO, 1977. Plus modeste, mais symptomatique, Matteo GribaldI Mofa écrivit un De methodo et ratione docendi (1541). Voir Quaglioni, 1999 et 2008. Sur la plupart de ces auteurs, voir Piano Mortari, 1991. 
reconnaissance d'un champ intellectuel nouveau), il avait commencé par se doter de véritables chartes pédagogiques: l'on pense aux traités de Leonardo Bruni ou à celui de Pier Paolo Vergerio au début du Xve ${ }^{\mathrm{e}}$ siècle ${ }^{43}$. Au XVI ${ }^{\mathrm{e}}$ siècle, on recommence à fonder une méthodologie de l'apprentissage juridique ${ }^{44}$. Le cœur du mouvement demeurait l'historicisation des sources et la systématisation du droit.

Pour les juristes humanistes, le droit romain n'était plus un horizon sans rivage, sans lieu ni date. Il convenait au contraire d'assigner une origine précise à chaque loi. Cette historicisation du corpus s'est opérée selon des modalités variées. Nous en évoquerons cinq, en signalant les effets sur la nature des sources ${ }^{45}$. 1) Les textes de la tradition juridique ont été rédigés à des périodes diverses. Ils ne constituent pas un bloc contemporain. Une fois la nécessité de dater les lois établie, il pouvait apparaître que des interventions législatives ultérieures avaient changé la loi; ceci explique les positions apparemment contradictoires de différentes lois citées dans le corpus. 2) Ce sont des juristes différents qui ont rédigé les textes. Or, ces juristes romains pouvaient s'opposer les uns aux autres: les divergences contenues dans le Digeste n'étaient pas qu'apparentes; elles étaient réelles. Par conséquent, les efforts de la scolastique universitaire pour faire tenir ensemble par la résolution des contraires des opinions divergentes n'avaient plus lieu d'être. 3) Les textes avaient été rédigés dans un contexte autre que celui pour lequel ils étaient maintenant cités ou allégués. Ceci apparaît justement grâce au travail philologique de restauration des inscriptiones: le corpus est un agrégat d'excerpta de différents juristes romains; les jointures ne sont pas visibles. Pourtant, on pouvait retrouver les Inscriptiones, c'est-à-dire la précision d'où était tiré le passage (auteur, titre de l'ouvrage, section). Des contextes intellectuels différents pouvaient donner lieu à des interprétations différentes. Il fallait en quelque sorte recontextualiser le corpus justinien pour chacune de ses lois. 4) Les textes avaient pu être corrompus. Il fallait donc comparer les manuscrits et les manuscrits grecs étaient mis à profit pour éclairer le sens des passages latins obscurs. C'était particulièrement net pour tous les cas où les négations grammaticales avaient « sauté » dans les copies des manuscrits. Cujas avec ses Adnotationes et emendationes était le maître incontestable de ces restitutions du texte originel. 5) Enfin, les textes pouvaient être interpolés. Justinien ou Tribonien avaient pu, pour les besoins de la cause, imposer des modifications à tout ou partie d'une loi ou d'un commentaire, sans en avertir quiconque. Il convenait donc de procéder à l'émendation du texte ${ }^{46}$.

Une telle méthodologie qui se met en place en France très progressivement avait des conséquences claires. Elle mettait évidemment en cause les tenants du mos italicus ${ }^{47}$. Les critiques se faisaient toujours plus nombreuses sur cette indifférence à la source même du droit. Il est vrai que des docteurs italiens des $\mathrm{XV}^{\mathrm{e}}$ et $\mathrm{XVI}^{\mathrm{e}}$ siècles avouaient

43. Voir Leonardo Bruni, De studiis et litteris (dans Bernard-Pradelle, éd., 2008, p. 549-612); Pier Paolo Vergerio, De ingenuis moribus ac liberalibus studiis (dans Woodward, 1896, ici 1996, p. 96-118).

44. Quaglioni, 1999; Cortese, 1992.

45. OSLER, 2001.

46. PalazZini FinetTi, 1953.

47. Sur la façon très subtile dont s'articulent les deux méthodes chez les meilleurs juristes, voir l'étude exemplaire de Minnucci, 2002, ainsi que Conte, 1993. 
ne pas toujours se préoccuper de la vérité du texte. Ainsi, un juriste aussi renommé que Carlo Ruini, nommé «doctor eminentis scientiae » à la « chaire de droit civil de l'après-midi ${ }^{48}$ ", à l'université de Bologne, rapporte, en 1511, que devant les efforts confus de certains de ses collègues siennois - qu'il appelle les Moderni Senenses (les Siennois modernes) - pour récupérer le sens des passages obscurs du Digeste en collationnant le manuscrit des Pandectes, il préfère lui se tourner vers les choses plus utiles: «[...] ad utiliora se convertere ${ }^{49}$. » Les utiliora sont les opinions avérées de ses prédécesseurs ${ }^{50}$.

\section{LES FONDEMENTS DE L'HUMANISME JURIDIQUE}

Pour autant, l'essentiel des critiques ne provient plus de milieux extérieurs à la science juridique, mais désormais (à la fin $\mathrm{Xv}^{\mathrm{e}}$ siècle) des juristes eux-mêmes. L'humanisme juridique est une réponse à la crise de crédibilité des juristes. En réalité, le mal était plus profond qu'une simple déficience philologique. C'est la place de ces juristes, jusqu'alors prompts à orienter les sentences judiciaires, notamment par le jeu des consilia, qui est remise en cause par le développement des tribunaux nationaux ou régionaux ${ }^{51}$ : nous citerons un passage du juriste et humaniste Nevizzano qui, dans sa Sylva nuptialis (1516), éclaire nettement cette crise de crédibilité du droit des docteurs, et la concurrence que leur livrent les cours souveraines :

« J'ai vu dans un procès de nobles trois ou quatre jugements conformes à la rigueur de la loi, et qui ont été par la suite jugés contraires par la cour souveraine (de Milan). Les pères (du sénat) ont l'habitude de dire qu'ils sont des juges en équité et non en

48. Il existait à Bologne, comme dans d'autres universités, des chaires du matin et celles de l'aprèsmidi. À Bologne, ces dernières étaient les plus prestigieuses et les mieux rémunérées.

49. RuINus, 1575 , p. 87b: « [...] omissa divisione, summario et casu, necnon quibsdam expositionibus ad istum textus secundum originalem literam Pandectarum relatam hic per aliquos, ex quo inferunt quaedam impossibilia, ut hic ostendunt Moderni Senenses, ad quos hic me refero, et ad utiliora me converto. » Voir CAVINA, 1998, p. 25.

50. Encore à la fin du XVI ${ }^{\mathrm{e}}$ siècle, il y a un goût prononcé et revendiqué pour le bartolisme au point que l'on crée des chaires de bartolisme dans les universités italiennes: voir MATTONE, 2007.

51. Pour un examen très précis de la façon dont les consultores étaient requis d'intervenir dans la pratique politique de la cité, voir TANZINI, 2007: ont été conservées, entre les années 1370 et 1415, des séries de Pareri dei Savi, requis par le notaire des annulations de condamnations (notaio di Camera) pour vérifier que les éventuelles annulations de condamnations ne lésaient pas les intérêts de la commune. Mais dès 1388, se profile une tension entre l'autorité législative de la ville, qui voit d'un mauvais œil le pouvoir " juridique » surveiller le pouvoir « législatif» et affirme que « $i$ facitori delle leggi debbano essere migliori interpreti che altri» (TANZINI, 2007, p. 162). La disparition des archives de consilia de ce type après 1415 empêche de juger de l'évolution du rôle des juristes florentins dans les procédures d'annulation des condamnations, mais une loi de 1412 interdisait l'annulation des condamnations sur la seule base des pareri des juristes et soumettait donc cette annulation à l'accord de la Signoria; il n'est donc pas exclu que le vide archivistique corresponde effectivement à une réduction de l'activité « consiliaire » des juristes florentins. En ce sens, Florence aurait été une des premières cités à avoir expérimenté la réduction du pouvoir des juristes dans le jeu institutionnel local. C'est à une conclusion voisine qu'était parvenu KIRSHNER, 1999, quand il évoquait la fin des consultorcrats florentins et les tentatives de la Seigneurie pour «politiser » les consilia. 
nullité. Et c'est pourquoi il faut féliciter ceux qui rédigent leurs sentences par écrit et les font imprimer pour les rendre connues à tous; de la sorte, toutes les parties sauront comment se comporter dans les procès et à quelles opinions se conformer ${ }^{52}$. "

Quelles que soient les allégations juridiques, aussi justes fussent-elles, le dernier mot n'est plus au docteur en droit, praticien du tribunal, mais au juge. Dans le fond, le droit jurisprudentiel des docteurs cède la place à une autre jurisprudence, celle des tribunaux suprêmes. Il est d'ailleurs frappant que, dans les universités italiennes, les efforts d'innovation visaient davantage à la création de chaires universitaires de haute technicité pratique qu'au financement de chaires d'histoire des Pandectes. Ainsi, l'université de Bologne créa-t-elle en 1509 une chaire de droit criminel, la première en Europe, bientôt suivie par des équivalents à Padoue, Ferrare, etc. ${ }^{53}$; et ce ne fut pas avant les années 1570 et 1580 que des chaires de jurisprudence humaniste, notamment sur les Pandectes, furent instituées dans ces mêmes universités italiennes ${ }^{54}$.

Quant à la notion de communis opinio doctorum (l'opinion commune des docteurs), elle a partie liée avec l'humanisme juridique: la notion a été élaborée au $\mathrm{XV}^{\mathrm{e}}$ siècle comme une tendance à réfréner la prolifération incontrôlable des consilia, comme une digue pour limiter les flots de la jurisprudence doctorale et comme un effort pour hiérarchiser les sources du droit. La loi des citations des souverains espagnols et portugais au $\mathrm{XV}^{\mathrm{e}}$ siècle ne dit pas autre chose qui prescrivait qu'en cas de doute sur les autorités, l'opinio Bartoli devait prévaloir sur les autres opinions. Mais il s'agissait toujours de rester dans les limites du ius commune ${ }^{55}$; les tentatives qui caractérisent l'humanisme juridique ne sont pas nécessairement différentes, en ce sens qu'elles ne portent pas atteinte au ius commune, mais qu'elles viennent plutôt enrichir les sources disponibles, car la grande affaire de l'humanisme juridique, c'est d'ouvrir les textes. Mario Ascheri a pu écrire qu'avec l'humanisme juridique, on passait du texte aux textes, de l'auctoritas à une pluralité d'auctoritates ${ }^{56}$. Les bartolistes œuvraient dans un système apparemment clos dans lequel ils exerçaient leur sagacité à créer une sorte d'hypertexte avec des éléments disséminés dans un corpus donné. Les juristes humanistes découvrent qu'il n'y a pas un droit romain, mais plusieurs, qu'il y a en outre un droit byzantin jusqu'alors soigneusement ignoré et qui s'avère riche en matière de droit public, qu'il y a aussi des droits nationaux (ce que les humanistes non juristes comme le Pogge avaient suggéré en leur temps), notamment coutumiers. Il n'est donc plus possible de se limiter aux sources léguées par les médiévaux. Il faut non seulement les amender, mais les amplifier. On pourrait toujours objecter à cette suggestion de Mario Ascheri que l'ouverture des sources ne modifie pas nécessairement le

52. NeVIzZANO, 1521, 1. V, chap. LXII, p. 554: «Vidi in causa nobilium de Casacho. in qua fuerunt latae tres vel quatuor sententiae conformer in rigore illius legis, fuisse postea contrarium iudicatum per summam curiam. Solent enim communiter illi patres illud dicere quod sunt iudices aequitatis et non nullitatis. Et propterea commendandi sunt qui decisiones suas ponunt in scriptis, et imprimi faciunt ut deveniant in notiatiam omnium, ut possint scire quomodo partes habeant in litibus se continere, et quibus opinionibus adhaere »; voir CAVINA, 1998.

53. GRENDLER, 2002 et 2009, p. 44-45.

54. Grendler, 2002, p. 451-470.

55. Costa, 1995.

56. AsCHERI, 1992. 
système juridique. En quoi rend-on la justice différemment $\mathrm{au} \mathrm{XVI} \mathrm{I}^{\mathrm{e}}$ siècle par rapport $\mathrm{au} \mathrm{XV}^{\mathrm{e}}$ siècle? Certes, les juristes humanistes revendiquaient l'axiome: «[...] bon texte, bonne justice; mauvais texte, mauvaise justice ${ }^{57}$. $\gg$ Mais, ne nous y trompons pas: on continue à publier et à vendre toujours plus d'ouvrages issus du mos italicus que du mos gallicus, et les éditions lyonnaises comme vénitiennes attestent de ce succès permanent de Bartole, Balde, Tartagni ${ }^{58}$, etc. Ce qui se modifie, c'est la place des juristes et non la nature du système juridique, ni le cursus universitaire en droit $^{59}$. En Italie, il n'est pas vraiment question de codification, de refondation des principes du droit sur la base de l'humanisme, mais davantage d'une réorganisation de l'ordre juridico-politique dans lequel les juristes se voient assigner une place différente ${ }^{60}$. En d'autres termes, le groupe des juristes, le plus souvent regroupés dans les collegia doctoraux ${ }^{61}$, qui constituait une figure sociale et institutionnelle dans le monde communal, a subi de profonds changements au cours du XVI ${ }^{\mathrm{e}}$ siècle: non pas parce que les juristes auraient été moins nombreux ou moins influents auprès des autorités politiques, mais parce que la nécessité intrinsèque de recourir à eux pour faire tenir les divers éléments du système politico-juridique de l'ordre communal (et même post-communal, si l'on se place au XV siècle) n'apparaissait plus aussi évidente. Dans les villes italiennes, le collège des docteurs en droit pouvait se trouver concurrencé par l'imposition sans discussion de principes législatifs qui réduisaient sa capacité interprétative; même une ville comme Bologne enregistre ce phénomène et ses juristes tentent $\mathrm{du} \mathrm{XVI}{ }^{\mathrm{e}}$ au XVIII ${ }^{\mathrm{e}}$ siècle de montrer que les statuts urbains sont assimilables à des éléments du droit naturel auxquels même le souverain (en l'occurrence le légat pontifical) ne pouvait déroger ${ }^{62}$. Mais la lutte était inégale, et quelle que soit la vitalité intellectuelle des facultés de droit ou des collèges de docteurs, ils devaient reconnaître que ce n'était plus par leur médiation (et leur intégration quasiment institutionnelle dans l'organigramme des pouvoirs urbains, comme aux plus beaux jours des cités communales) que se résolvaient les problèmes «constitutionnels » de la ville. À ce titre, la rénovation des textes sous la bannière humaniste n'apportait rien de substantiel au groupe social des juristes italiens. De là vraisemblablement, la portée si fortement différenciée du mouvement entre la France et l'Italie!

Un point important unit néanmoins les différentes facettes de cet humanisme, par ailleurs nuancé entre les revendications extrêmes d'un Hotman qui en vient à rejeter le droit romain comme inutile en France ${ }^{63}$ et celles nettement plus modérées d'un André Alciat. Ce point commun, semble-t-il, correspond à la place des pouvoirs normatifs dans la hiérarchie des droits: l'ouverture voulue des sources du droit nécessitait une clé de voûte, garante du système. Alciat, par exemple, voudrait réduire la liberté

57. TROJE, 1970, p. 40.

58. OSLER, 2001.

59. BIROCCHI, 2007.

60. Pour les modifications françaises de cette perspective, voir Krynen, 2000, et surtout FioraVAnTI, 2002, sur « l'état juridictionnel ».

61. Sur l'importance des collèges de docteurs en Italie, voir Gilli, 2005, et Brambilla, 2007; pour sa valeur exemplaire, Zorzoli, 1986.

62. BeNEDICTIS, 2008.

63. Singulièrement dans son Anti-Tribonien : voir Hotman, 1998. 
d'interprétation et introduire une sorte de rationalité humaniste sous la direction du Prince, nouveau Justinien qui limiterait les jeux d'interprétation ${ }^{64}$, en particulier en matière de consilia (avis juridiques des docteurs en droit). Il témoigne ainsi, encore, d'une insatisfaction devant l'excès et appelle, en même temps, à une réorganisation juridique. Il désire donc clairement une prédominance du législateur sur le système des sources et la pratique judiciaire. N'est-ce pas ce que Hotman augure lui aussi en plaçant la France et sa législation hors de l'orbe juridique romain?

On voit bien combien la quête fondamentale de la hiérarchie des sources ne peut que renvoyer à la nature des pouvoirs politiques et à celle de la hiérarchie culturelle. Lorsque dans les années 1440, Lorenzo Valla avouait son admiration pour le droit romain et son mépris des juristes, il liait la gloire de ce corpus au destin de la langue latine (dans la quatrième préface aux Elégances de la langue latine, il s'exclame: « una lex, una est lingua romana $\left.{ }^{65} »\right)$. Quelques décennies plus tard, les juristes humanistes français lui répondaient, textes à l'appui, que l'on pouvait redimensionner l'influence du droit impérial en montrant ses imperfections originelles et ses carences et qu'il y avait bel et bien une disjonction entre langue et droit. La restauration de la pureté du monument justinien n'allait pas se faire sous le signe de l'unité et du primat de l'Italie. Peut-être est-ce là un point insuffisamment mis en exergue par la recherche. Mieux encore, à voir avec quelle fougue une partie des intellectuels français, puis européens, se lancent dans la recherche d'une meilleure édition du texte, on ne peut s'empêcher de penser que le terme humaniste sous lequel on subsume ce courant ne vise à rien moins qu'à « dé-boloniser » ou " dé-italianiser » le droit commun. Dans le fond, que la critique prenne la forme d'une approche historique, à la façon d'un Budé, ou d'une dénonciation de la barbarie justinienne, à la façon d'un Hotman, c'est la tradition italienne du droit qui est visée et l'enfermement épistémologique de la sciencia legalis dans les limites imposées par les juristes italiens. Car, par ailleurs, quels changements l'humanisme apporte-t-il? La façon d'interpréter les textes est-elle substantiellement modifiée? Plus encore, l'usage d'un texte non corrompu apporte-t-il un surcroît de protection aux justiciables? L'herméneutique juridique est-elle vraiment changée?

$\mathrm{Au}$ début $\mathrm{du} \mathrm{XIV}^{\mathrm{e}}$ siècle, Iacopo Bottrigari déjà rapportait que les juges français avaient l'habitude de justifier leurs sentences par la ratio iuris (raison du droit) et non par les textes ${ }^{66}$. Au milieu du XVI ${ }^{\mathrm{e}}$ siècle, Cujas, alors même qu'il introduit ses Observations et émendations du Digeste affirme, de manière claire: « [...] sed plus ipse iuris rationi tribuo, quam illi scripturae, quia quidem, nulla res est quae quidem facilius depravari est ${ }^{67}$. $\gg$ Assurément retrouver la raison du droit est plus facile sur un texte authentique ou clairement contextualisé que sur un texte malléable parce qu'incertain. Cette importance de la ratio iuris que le juriste est à même d'extraire des lois sans être entravé par elles ne vient-elle pas limiter la portée de la mutation humaniste

64. Alciat, 1560, p. 283 r ${ }^{\circ}$.

65. «Une seule loi, une seule langue romaine. »

66. Cité par CoRTese, 1995, p. 456.

67. «Mais moi j'accorde plus à la raison du droit qu'eux au texte, parce qu'il n'y a rien de plus facile que de le corrompre. » 
du droit? Par le jeu de l'historicisation, les humanistes portaient un coup fatal à la sacralité autoproclamée des lois romaines. Cette profondeur chronologique retrouvée aboutissait à l'affirmation d'une science juridique qui n'entendait plus se soumettre à l'autorité d'un texte étranger autosuffisant - et ce, au moment même où s'affirmait l'activité législative et normative du monarque français. Ce que les docteurs en droit italiens perdaient avec cette méthodologie, c'est moins la maîtrise d'une auctoritas que la fluidité d'interprétation que ces sources leur permettaient, grâce à laquelle ils ne cessèrent entre le $\mathrm{XIII}^{\mathrm{e}}$ siècle et le $\mathrm{XV}^{\mathrm{e}}$ siècle, d'occuper une place centrale dans les cités ou les régimes seigneuriaux ${ }^{68}$. Que leur restait-il comme créativité juridique s'il apparaissait que les subtiles distinctions, dubia, summae et autres divisiones ${ }^{69}$ sur lesquelles ils faisaient reposer leur science interprétative des contradictions du corpus iuris n'étaient que le produit historique empirique d'une compilation mal ficelée et non la projection d'un ordonnancement subtil qu'il leur revenait d'éclairer? On comprend mieux l'essor de la littérature des decisiones judiciaires qui, sans prétendre à autre chose qu'à aider la pratique ordinaire, s'avéraient plus utiles à l'étudiant que les multiples commentaires des lois ${ }^{70}$. Peut-être les juristes italiens n'ont-ils pas perçu combien cette nouvelle méthodologie remettait certes en jeu leur position sociale, mais pouvait aussi leur apporter un surcroît de prestige par le rôle central que le juriste assumait dans les nouveaux savoirs ${ }^{71}$ : le juriste se faisait philologue, historien, voire philosophe du droit, et pouvait ainsi récupérer dans le champ culturel tout entier ce qu'il perdait dans le seul secteur juridique. De façon presque anecdoctique, c'est ce que tenta de faire Barthélémy de Chasseneuz, en 1529, dans son Catalogus gloriae Mundi ${ }^{72}$ qui reprend, assurément, l'héritage accursien du droit comme vera philosophia. Toutefois, il établit le lien avec les esprits humanistes, comme Pic de la Mirandole, pour reconstruire une histoire de la formation de l'homme de savoir où les arts libéraux collaborent activement (mais, bien sûr, de façon subordonnée) avec la science juridique, pour aboutir à l'ultime avatar de l'homme de la Renaissance: le jurisconsultus summa cum laude (jurisconsulte digne du plus grand éloge), synthèse harmonieuse de la vie active et de la vie contemplative. Un tel programme d'autocélébration (qui a bénéficié d'un réel engouement du public, si l'on juge par les rééditions de l'ouvrage jusqu'au XVIII ${ }^{\mathrm{e}}$ siècle) prêterait à sourire, s'il n'était le témoignage d'une volonté des juristes de relever le défi des humanistes, en se plaçant aussi sur le terrain d'une collaboration des disciplines, sous l'égide du droit.

Pour n'avoir pas voulu suivre cette voie, une grande partie de la science légale italienne de la première modernité s'est trouvée intellectuellement déclassée, laissant

68. Parmi une vaste littérature sur les liens des juristes et du droit justinien, voir AscHERI, 1996 (en ligne sur Reti Medievali); voir aussi les pages que consacre Grossi, 2002, p. 162-175.

69. Les doutes (dubia), les sommes (summae), les divisions du texte (divisiones) forment quelquesuns des éléments de l'exégèse juridique à l'université.

70. À titre de comparaison, signalons que l'Espagne a vu fleurir aussi au XVI ${ }^{\mathrm{e}}$ siècle un nouveau genre de littérature juridique, les prácticas, rédigées par des letrados non universitaires, qui se targuaient de rendre de plus avérés services à leurs lecteurs et aux justiciables que les ouvrages de pure doctrine, tout en manifestant une ferme volonté de servir le droit royal. Voir CAPOROSSI, 2009, notamment p. 76.

71. Voir Kelley, 1997.

72. Sur le personnage, outre l'article ci-dessus, voir LeYte, 2003. 
au mos gallicus le loisir de capter l'esprit du siècle. C'est un peu comme si le projet de Pétrarque, certes demeuré très imprécis ${ }^{73}$, n'avait pas donné les fruits escomptés en Italie, mais chez le pays voisin et rival. Assurément le bartolisme a vécu encore de beaux jours en Italie, comme en Europe, au XVI ${ }^{\mathrm{e}}$ siècle ${ }^{74}$, et l'humanisme juridique a souvent été plus une manière d'enseigner, voire un supplément d'âme pour juristes, qu'une refonte heuristique du droit, du moins dans la première moitié du siècle. Mais, pour autant, ce serait une erreur de penser, comme le fait Douglas Osler, que l'aura dont est entouré le mouvement n'est due qu'à une reconstruction historiographique des $\mathrm{XVIII}^{\mathrm{e}}$ et $\mathrm{XIX}^{\mathrm{e}}$ siècles, élaborée par des juristes humanistes pour faire de l'humanisme juridique le pivot de la science du droit à l'époque moderne, c'est-àdire à leur propre époque ${ }^{75}$. Si les juristes italiens de l'époque moderne ont moins influencé le droit européen de leur temps que leurs prédécesseurs, c'est parce que la fonction quasi oraculaire des doctores legum, à travers les consilia, et l'intégration ex officio (c'est-à-dire en tant que docteurs en droit) dans les conseils urbains, avait disparu au moment où les cours souveraines un peu partout en Europe étaient devenues les véritables dépositaires de l'ordre juridique voulu par les monarchies; c'est aussi et surtout que la (quasi) co-extensivité médiévale de la notion de jurisdictio et de celle de pouvoir politique s'était estompée pour laisser apparaître dans l'Europe moderne une fonction publique administrative, dont la multiplication des tribunaux et des offices était le signe, qui nécessitait d'être pensée à nouveaux frais ${ }^{76}$. Le déclassement des doctores legum au profit des juges et des parlementaires a été de pair avec la naissance de l'humanisme juridique, en France ${ }^{77}$ comme en Italie. Comment ne pas voir que cette concomitance n'est pas fortuite, mais qu'elle porte témoignage de la crise de ce droit jurisprudentiel des docteurs qui avait été la marque de fabrique du Moyen Âge italien. Désormais, le branle dans la réflexion politique n'était plus donné par des légistes ex officio ${ }^{78}$. Machiavel, Guichardin, avaient tous deux fait des études juridiques, mais ce n'est pas en tant que juristes qu'ils se sont fait connaître. Botero était, de son côté, théologien et ne déployait pas ses critiques dans un horizon juridico-politique, mais dans celui de la tradition aristotélicienne ${ }^{79}$. En Europe même, les grands systèmes juridiques issus de la dynamique humaniste sont nés d'esprits peu liés à l'Université (si l'on met à part le cas, somme toute bref, de Bourges): Bodin, Hotman, Althusius, Grotius, par exemple ${ }^{80}$. L'évolution technique de la jurisprudentia cultior pouvait s'avérer d'une grande beauté pour les arabesques de la raison historique, elle ne semble pas avoir eu d'impact sur la culture

73. MANZIN, 1994.

74. CARavale, 2005.

75. OSLER, 2001.

76. MANNORI, 1990.

77. Qu'il suffise de rappeler que lors de la création du parlement de Toulouse en 1443, les membres étaient presque tous docteurs de l'Université et qu'en 1610, entrait au parlement le dernier membre à être issu de l'Université. La rupture entre l'Université et le Palais était consommée. Voir l'introduction de Jean-Paul Pittion dans PitTion, éd., 2009, p. 13.

78. SCATtola, 2003.

79. À titre d'exemple, voir Borrelli, 1995.

80. Villey, 2003, p. 477-479. 
politique italienne du temps ${ }^{81}$. Il faudra d'ailleurs attendre le XVIII ${ }^{\mathrm{e}}$ siècle pour que les facultés de droit italiennes s'ouvrent à la science du droit public, ce fruit tardif de l'humanisme juridique ${ }^{82}$.

\section{LISTES DES RÉFÉRENCES}

\section{I- Sources}

Alciat (André), 1560, De verborum significatione, dans ID., Opera omnia, Lyon, S. Glyphe.

Ferrer (Joan Ramon), 2006, De antiquitate legum, éd. Antoni CoBos, Bellcaire d'Empordà, Edicions Vitel.

Hotman (François), 1998, Antitribonian, ou Discours d'un grand et renommé iurisconsulte de nostre temps sur l'estude des loix, Saint-Étienne, Presses universitaires de Saint-Étienne.

Nevizzano (Giovanni), 1521, Sylva nuptialis, Paris, Impressa Lugduni per Joannem Moulin, alias de Cambray.

Pétrarque, 1943, Rerum memorandarum libri, éd Giuseppe Billanovich, Florence, Sanzoni (Edizione nazionale delle opere di Petrarca, $\mathrm{n}^{\circ} 14$ ).

Ruinus (Carolus), 1575, In secundam Infortiati partem Commentaria, Bononiae, Apud Societatem Typographiae Bononiensis.

\section{II-Études}

Arabeyre (Patrick), 1999, « Droits et histoire: les fondements de la règle de succession au royaume de France chez Guillaume Benoît (1455-1516) », dans KrYNEN, éd., 1999, p. $125-154$

Arabeyre (P.), 2003, Les Idées politiques à Toulouse à la veille de la Réforme, Toulouse, Presses de l'université des sciences sociales.

Arabeyre (P.), 2008, «Un enseignement de science politique dans les facultés de droit canonique françaises de la fin du Xv $v^{\mathrm{e}}$ au début du XvI siècle (Paris, Cahors, Toulouse) », dans Krynen et Stolleis, éd., 2008, p. 125-154.

Ascheri (Mario), 1977, "Giuristi, umanisti e istituzioni del Tre-Quattrocento: qualche problema », Annali del Istituto storico italo-germanico in Trento, vol. III, p. 43-73.

AsCHERI (M.), 1992, « I giuristi, l'umanesimo e il sistema giuridico dal medioevo all'età moderna », El dret comu'i Catalunya, Barcelone, Estudis Fundació Noguera, p. 145-166.

AsCherI (M.), 1996, « Il "dottore" e lo statuto: una difesa interessata? », Rivista di storia del diritto italiano, vol. LXIX, p. 95-113.

81. À cet égard, le cas français est sensiblement différent, car si l'on met à part les travaux savants d'un Cujas ou d'un Budé, un des effets les plus remarquables de l'humanisme juridique français fut précisément de fournir un nouveau cadre dogmatique et systémique propice à la construction des droits nationaux et à l'esprit de codification juridique: tel est le sens des œuvres, par ailleurs divergentes entre elles, de Charles Dumoulin, de François Hotman ou de Jean Bodin. Rien de comparable avec le « cultisme » italien du XvI ${ }^{\mathrm{e}}$ siècle dont le développement n'a fécondé ni les pratiques judiciaires ni la réflexion politique: voir, par exemple, PIANo MORTARI, 1965. Il n'est qu'à voir également comment Bodin regardera d'un œil très critique la tentative jugée aberrante de Bartole d'établir à partir du droit romain des principes universels de jurisprudence (voir SKINNER, 2001, p. 746). Sur la systémique juridique du XVI e siècle, voir VILley, 2003, "La philosophie de l'humanisme et ses composantes », p. 381-394 (rappelons que cet ouvrage est issu de cours, souvent anciens, de l'auteur).

82. Renzo Villata, 2008. 
AsCherI (M.), 1998, « Poliziano filologo del diritto tra rinnovamento della giurisprudenza e della politica », dans Fera (Vincenzo) et Martelli (Mario), éd., Agnolo Poliziano poeta scrittore, filologo, Florence, Olschki, p. 323-331.

Benedictis (Angela de), 2008, «Iura municipalia e jus publicum bononiense nello Studio di Bologna », dans KRYNEN et STolleis, éd., 2008, p. 529-549.

Bernard-Pradelle (Laurence), éd., 2008, Leonardo Bruni Aretino. Histoire, éloquence et poésie à Florence au début du Quattrocento, Paris, Champion.

BIrocCHI (Italo), 2007, « Contenuti e metodi dell'insegnamento : il diritto nei secoli XVI-XVIII », dans Brizzi (Gian Paolo), Del Negro (Piero) et Romano (Andrea), éd., Storia delle università italiane, Messine, Sicania, vol. II, p. 243-269.

Black (Robert), 2001, Humanism and Education in Medieval and Renaissance Italy. Tradition and Innovation in Latin Schools from the Twelfth to the Fifteenth Century, Cambridge, Cambridge University Press.

BorRelli (Gianfranco), 1995, «Aristotelismo politico e ragion di stato in Italia », dans BALDINI (A. Enzo), éd., Aristotelismo politico e ragion di stato, Florence, Olschki, p. 181-199.

Botтin (Francesco), 1982, «La polemica umanistica contro la scolastica: l'origine di un topos storiografico », dans ID., La Scienza degli occamisti. La scienza tardo-medievale dalle origini del paradigma nominalista alla rivoluzione scientifica, Rimini, Maggioli, p. 277-313.

Brambilla (Elena), 2007, « Collegi dei dottori universitari e collegi professionali », dans Brizzı (Gian Paolo), Del Negro (Piero) et Romano (Andrea), éd., Storia delle università italiane, Messine, Sicania, vol. II, p. 303-345.

CAPOROSSI (Olivier), 2009, « Les livres de pratique judiciaire castillans, XVI $-\mathrm{XVII}^{\mathrm{e}}$ siècles ", dans Pittion, éd., 2009, p. 59-76.

CAPRIOLI (Severino), 1969, Indagini sul Bolognini. Giurisprudenza e filologia nel Quattrocento italiano, Milan, Giuffré.

Caravale (Mario), 2005, Alle origini del diritto europeo. Ius commune, droit commun, Common Law nella dottrina giuridica della prima età moderna, Bologne, Monduzzi.

CARPINTERo (Francisco), 1977, «"Mos italicus”, "mos gallicus” y el humanismo racionalista. Una contribución a la historia de la metodología jurídica», Ius Commune, nº 6, p. 108-171.

Cavina (Marco), 1998, Carlo Ruini. Una autorità del diritto comune fra Reggio Emilia e Bologna, fra XV e XVI secolo, Milan, Giuffré.

CONTE (Emanuele), 1993, « Umanisti e "bartolisti” tra i colleghi romani di Marc Antoine Muret », Rivista internazionale di diritto comune, $\mathrm{n}^{\circ}$ 4, p. 171-189.

CORTESE (Ennio), 1982, "Legisti, canonisti e feudisti: la formazione di un ceto medievale», dans Università e società nei secoli XII-XVI. Centro ital. di studi di storia e d'arte di Pistoia. Atti del nono convegno... 1979, Pistoia, Centro ital. di studi di storia e d'arte di Pistoia, p. 195-281.

Cortese (E.), 1985, « Sulla scienza giuridica a Napoli tra Quattro e Cinquecento », dans Scuole diritto e società nel mezzogiorno medievale d'Italia, Catania, Tringale, vol. I, p. 131-134.

Cortese (E.), 1992, «Tra glossa, commento e umanesimo », Studi senesi, n 104, p. 458-503, rééd. dans Garcia y Garcia (Antonio) et Weimar (Peter), éd., Miscellanea Domenico Maffei dicata, Historia-Jus-Studium, Keip, Goldbach,1995, vol. III, p. 29-74.

Cortese (E.), 1995, Il Diritto nella storia medievale, Rome, Il Cigno, 2 vol.

Costa (Pietro), 1995, «"Ius commune”, "ius proprium”, "interpretatio doctorum": ipotesi per una discussione », dans Iglesia Ferreirós (Aquilino), éd., El dret comú i Catalunya, Atti del IV simposio internazionale, Barcellona, 27-28 maggio 1994, Barcelone, Estudis Fundació Noguera, p. 29-42. 
ERrera (Andrea), 2003, Il Concetto di scientia iuris dal XII al XIV secolo. Il ruolo della logica platonica e aristotelica nelle scuole giuridiche medievali, Milan, Giuffré.

FERRARY (Jean-Louis), 1995, « Naissance d'un aspect de la recherche antiquaire. Les premiers travaux sur les lois romaines de l'Epistula ad Cornelium de Filelfo à l'Historia juris civilis d'Aymar du Rivail », dans Crawford (Michael A.) et Ligota (Christopher R.), éd., Ancient History and the Antiquarian. Essays in Memory of Arnaldo Momigliano, Londres, Warburg Institute, p. 33-71.

Fioravanti (Maurizio), 2002, « Stato e costituzione », dans ID., éd., Lo Stato moderno in Europa : istituzioni e diritto, Rome/Bari, Laterza, p. 3-36.

FubINI (Riccardo), 1990, «Lorenzo Valla tra il Concilio di Basilea e quello di Firenze, e il processo dell'Inquisizione ", dans Conciliarismo, stati nazionali, inizi dell'umanesimo, Atti del 25 convegno storico internazionale dell'Accademia tudertina e del Centro di studi sulla spiritualità medievale, Todi, 9-12 ottobre 1988, Spolète, Centro italiano di studi sull'Alto Medioevo, p. 287-318.

Fubini (R.), 1991, «Contestazioni quattrocentesche della Donazione di Costantino: Niccolò Cusano, Lorenzo Valla ", Medioevo e rinascimento. Annuario del Dipartimento di studi sul Medioevo e il Rinascimento dell' Universita di Firenze, ${ }^{\circ}{ }^{2}$, p. 19-61.

Gagliano (Marina), 2007, «Prophétisme et polémique dans les sonnets babyloniens et les épîtres Sine nomine de Pétrarque », Arzanà. Cahiers de littérature médiévale italienne, $\mathrm{n}^{\circ} 12$, p. $137-152$.

GARANCINI (Gianfranco), 1990, « La coutume dans les droits italiens du bas Moyen Âge », dans La Coutume - the Custom. Recueil de la société Jean Bodin, Bruxelles, Éditions de la librairie encyclopédique, p. 132-134.

Garin (Eugenio), 1947, La Disputa delle arti nel '400, Florence, Vallechi.

Gilli (Patrick), 1998, «Le conflit entre le juriste et l'orateur d'après une lettre de Cosma Raimondi, humaniste italien en Avignon (c. 1431-1432) », Rhetorica. Journal of the History of Rhetoric, vol. XVI, p. 259-286.

Gilli (P.), 2003, La Noblesse du droit. Débats et controverses sur la culture juridique et la place des juristes dans l'Italie médiévale, Paris, Champion.

Gilli (P.), 2005, «Les collèges de juristes en Italie centro-septentrionale au XV siècle: autorité doctorale et contrôle social », dans Kouamé (Thierry), AtTal (Frédéric) et Garrigue (Jean), éd., Les Universités en Europe du XIII siècle à nos jours. Espaces, modèles et fonctions, Paris, Publications de la Sorbonne, p. 113-130.

Gilli (P.), 2007, «Disciplines académiques et modernité des savoirs: le choix d'une politique culturelle à Florence (fin XIV ${ }^{\mathrm{e}}$-milieu XV $\mathrm{XV}^{\mathrm{e}}$ siècle) », dans GILLI (Patrick), LE BLÉVEC (Daniel) et Verger (Jacques), éd., Les Universités et la ville au Moyen Âge. Cohabitation et tension, Leyde, Brill, p. 327-340.

Gilli (P.), 2008, "Les Pandectes pisanes: fortunes et infortunes d'un texte au Moyen Âge », dans ID., éd., Les Élites lettrées au Moyen Âge, Montpellier, Presses universitaires de la Méditerranée, p. 231-246.

GioRDANENGo (Gérard), 1999, « Jus commune et “droit commun” en France du XIII au XV" siècle », dans Krynen, éd., 1999, p. 219-247.

Grendler (Paul), 2002, The Universities of the Italian Renaissance, Baltimore/Londres, John Hopkins University Press.

Grendler (P.), 2009, « Continuity and Change in Italian Universities between the Middle Ages and the Renaissance », dans EIsenblicher (Konrad), éd., Renaissance Medievalism, Toronto, Center for Reformation and Renaissance Studies, p. 33-51.

Grossi (Paolo), 2002, L’Ordine giuridico medievale, Rome/Bari, Laterza. 
KelLey (Donald), 1997, « Jurisconsultus perfectus: the Lawyer as Renaissance Man », dans ID., The Writing of History and the Study of Law, Aldershot, art. $\mathrm{n}^{\circ} 13$.

KIRSHNER (Julius), 1999, «Consilia as Authority in Late Medieval Italy: the Case of Florence », dans Ascheri (Mario), Baumgärtner (Ingrid) et Kirshner (Julius), éd., Legal Consulting in the Civil Law Tradition, Berkeley, The Robbins Collection, p. 107-142.

KRYNEN (Jacques), éd., 1999, Droit romain, jus civile et droit français, $\mathrm{n}^{\circ}$ spéc. des Études d'histoire du droit et des idées politiques ( $\left.\mathrm{n}^{\circ} 3\right)$, Toulouse, Publications de la faculté de droit.

KRYNEn (J.), 2000, «Entre science juridique et dirigisme: le glas médiéval de la coutume», Cahiers de recherches médiévales, $\mathrm{n}^{\circ} 7$ (http://crm.revues.org/document892.html).

Krynen (Jacques) et Stolleis (Michael), éd., 2008, Science politique et droit public dans les facultés de droit européennes (XIII ${ }^{e}$-XVIII ${ }^{e}$ siècle), Francfort-sur-le-Main, Klosterman.

LaUREYs (Marc), 1995, «At the Threshold of Humanist Jurisprudence: Andrea Fiocchi's De potestatibus Romanis », Bulletin de l'Institut historique belge de Rome, $\mathrm{n}^{\circ}$ 65, p. 25-42.

Leyte (Guillaume), 2003, « Le monde et sa représentation d'après le Catalogus glorice mundi de Barthélemy de Chasseneuz », dans Le Concept de représentation dans la pensée politique, Aix-en-Provence, Presses universitaires d'Aix-Marseille, p. 33-44.

Lines (David A.), 2006, « Humanism and the Italian Universities », dans Celenza (Christopher) et Gouwens (Kenneth), éd., Humanism and Creativity. Essays in Honor of Ronald Witt, Leyde, Brill, p. 327-346.

MAfFer (Domenico), 1972, Gli inizi dell'umanesimo giuridico, $2^{\mathrm{e}}$ éd., Milan, Giuffré.

MAnNori (Luca), 1990, « Per una preistoria della funzione amministrativa. Cultura giuridica e attività dei pubblici apparati nell'età del tado diritto comune », Quaderni fiorentini per la storia del pensiero giuridico moderno, $\mathrm{n}^{\circ} 19$, p. 322-504.

Manzin (Maurizio), 1994, Il Petrarchismo giuridico. Filosofia e logica del diritto agli inizi del umanesimo, Padoue, Cedam.

Marsh (David), 1980, Quattrocento Dialogue: Classical Tradition and Humanist Innovation, Harvard, Harvard University Press.

Mattone (Antonello), 2007, « Manuale giuridico e insegnamento del diritto nelle università italiane del XVI secolo»,Diritto@storia, n6 (http://www.dirittoestoria.it/6/Contributi/ Mattone-Manuale-giuridico-insegnamento-universit-XVI-secolo.htm).

MAYALI (Laurent), 1999, « Ius civile et Ius commune dans la tradition juridique médiévale », dans KRYNEN (Jacques), éd., 1999, p. 201-217.

Minnucci (Giovanni), 2002, Alberico Gentili tra mos italicus $e$ mos gallicus. L'inedito commentario Ad legem Juliam de adulteriis, Bologne, Monduzzi.

Orestano (Riccardo), 1987, Introduzione allo studio storico del diritto romano, Bologne, Il Mulino.

Osler (Douglas J.), 1990, « Filippo Beroaldo e l'umanesimo giuridico », dans Sapere elè potere. Discipline, dispute e professioni nell'Università medievale e moderna. Il caso bolognese a confronto. Atti del convegno, Bologna, 13-15 Aprile 1989, Bologne, Istituto per la storia di Bologna, vol. I, p. 233-241.

OsLer (D. J.), 2001, « Images of legal Humanism », Surfaces, vol. IX (http://www.pum.umontreal.ca/revues/surfaces/vol9/osler.htm).

Palazzini Finetti (Luigi), 1953, Storia della ricerca delle interpolazioni nel corpus iuris Giustiniane, Milan, Giuffré.

PAternoster (Annick), 2000, « La politesse positive dans le dialogue de la Renaissance italienne ( $\mathrm{XV}^{\mathrm{e}}-\mathrm{XVI}^{\mathrm{e}}$ siècle) ou l'art de doser les compliments », dans Wauthion (Michel) et Simon (Anne-Catherine), éd., Politesse et idéologie. Rencontres de pragmatique et de rhétorique conversationnelles, Louvain, Peeters, p. 303-311. 
Piano Mortari (Vincenzo), 1965, « La sistematica come ideale umanistico dell'opera di Francesco Connano », dans Studi in onore di Gaetano Zingali, Milan, Giuffré, vol. III, p. 557-573.

Piano Mortari (V.), 1991, Itinera juris. Studi di storia giuridica dell'etá moderna, Naples, Jovene.

PitTion (Jean-Paul), éd., 2009, Droits et justice dans l'Europe de la Renaissance, Paris, Champion.

Quaglioni (Diego), 1984, Pietro del Monte a Roma. La tradizione del «Repertorium utriusque iuris » (c. 1453). Genesi de diffusione della letteratura giuridico-politica in età umanistica, Rome, Edizioni dell'Ateneo.

Quaglioni (D.), 1999, «Tra bartolisti e antibartolisti: l'Umanesimo giuridico e la tradizione italiana nella Methodus di Matteo Gribaldi Mofa (1541) », dans LiotTa (Filippo), éd., Studi di storia del diritto medioevale e moderno, Bologne, Monduzzi, p. 185-212.

Quaglioni (D.), 2000, « Primi appunti per un commento al "De iure" di Leon Battista Alberti », Albertiana/Societé internationale Leon Battista Alberti, n ${ }^{\circ}$, p. 201-219.

Quaglioni (D.), 2004, «Il contributo della canonistica al primo umanesimo: il "De iure" di Leon Battista Alberti (1437) », dans GILLI (Patrick), éd., Humanisme et Église en Italie et en France méridionale, Rome, École française de Rome, p. 187-199.

Quaglioni (D.), 2008, « L'éducation du juriste face au pouvoir: la Methodus de Matteo Gribaldi Mofa (1541) », dans Krynen et Stolleis, éd., 2008, p. 347-357.

Rigaudière (Albert), 2004, "Un rêve royal français: l'unification du droit», C-R.A.I.B.L. (http://www.aibl.fr/fr/seance/discours/disc_rigaudiere.html).

Renzo Villata (Maria Gigliola di), 2008, «Le droit public en Lombardie au XVIII ${ }^{\mathrm{e}}$ siècle et l'Europe », dans Krynen et Stolleis, éd., 2008, p. 583-612.

Rossi (Giovanni), 1996, Duplex est usufructus. Ricerche sulla natura dell'usufrutto nel diritto comune, II: Da Baldo agli inizi dell'umanesimo giuridico, Milan, Giuffré.

Rossi (G.), 1999, «Un umanista di fronte al diritto: a proposito del "De iure" di Leon Battista Alberti », Rivista di storia del diritto italiano, vol. LXXII, p. 77-154.

RundLE (David), 2004, «The Book-Collecting of Paul II and his contemporaries », dans GiLLI (Patrick), éd., Humanisme et Église en Italie et en France méridionale, Rome, École française de Rome, p. 178-185.

SAVElli (Rodolfo), 1994, "Tribunali, Decisiones e giuristi. Proposta per un ritorno alle fonti », dans Chittolini (Giorgio), Molno (Anthony) et Schiera (Paolo), éd., Origini dello Stato. Processi di formazione statale in Italia fra medioevo ed età moderna, Bologne, Il Mulino, p. 397-421.

Scattola (Merio), 2003, Dalla virtù alla scienza. La fondazione e la trasformazione della disciplina politica nell'età moderna, Milan, Franco Angeli.

SkInNER (Quentin), 2001, Les Fondements de la pensée politique moderne, trad. franç. Jean-Yves Poullloux, Paris, Albin Michel.

TANZINI (Lorenzo), 2007, Il Governo delle leggi. Norme e pratiche delle istituzioni a Firenze dalla fine del Duecento al inizio del Quattrocento, Florence, Edifir.

Thireau (Jean-Louis), 2003, «Humanisme [jurisprudence]», dans Alland (Denis) et Rials (Stéphane), éd., Dictionnaire de la culture juridique, Paris, Presses universitaires de France, p. $795-800$.

TroJe (Hans Erich), 1970, «Die europaïsche Rechtsliteratur under dem Einfluss des Humanismus ", Ius Commune, Veröffentlichungen des Max-Planck-Instituts für Europäische Rechtsgeschichte, $\mathrm{n}^{\circ}$ 3, p. 33-63. 
VILley (Michel), 2003, Histoire de la pensée juridique moderne, Paris, Presses universitaires de France.

WitT (Ronald), 2000, In the Footsteps of the Ancients. The Origins of Humanism from Lovato to Bruni, Leyde, Brill.

WitT (R.), 2008, « The Early Communal Historians, Forerunners of the Italian Humanist », dans Eisenbichler (Konrad) et Terpstra (Nicholas), éd., The Renaissance in the Streets, Schools, and Studies. Essays in Honour of Paul F. Grendler, Toronto, Centre for Reformation and Renaissance Studies, p. 103-124.

Woodward (William H.), 1896, Vittorino da Feltre and Other Humanist Educators, Cambridge, rééd. Toronto, University of Toronto Press, 1996.

Zorzoli (Maria Carla), 1986, Università, dottori, giureconsulti. L'organizzazione della « Facoltà legale » di Pavia nell'età spagnola, Padoue, Cedam. 